medium, so dense that there are very tnany molecules per cubic wave-length-for example, in the atmo. sphere-must arise from the thermal motions of the molecules rather than from irregularity of their spacing. In directions, however, that are nearly coincident with the transmitted ray there can be no sensible dispersal of phase from either cause; disturbances, therefore, completely conspire, and the light scattered by the molecules in such directions is, in Lord Rayleigh's phrase, specially favoured. Prof. Raman points out that if the phases in directions near that of the ray did not in fact thus agree, the molecules of the material medium could take no concordant part in the transmission of the energy of the main beam, and regular propagation would be impossible. It is involved in this remark that each molecule will exert its full effect on the index of refraction, however irregular the distribution may be, provided it is not so dense that the molecules will obstruct each other; and, moreover, the thermal motions will not disturb this
INDIAN ASTRONOMICAL INSTRUMENTS."

INDIAN astronomy, handed down to us in a 1 series of text-books, the Siddhântas, of which the earliest dates from about A.D. 40o, is an offspring of Greek astronomy. Via Babylon and the Greek kingdom of Bactria (the Kabul valley and the Punjab), Greek science was introduced into India in the course of the two or three centuries following the invasion of Alexander the Great. While, during the Middle Ages, many astronomers in western Asia and North Africa did good work by re-determining astronomical constants and improving planetary tables by new observations, no attempts whatever in this direction were made in India. It is therefore very curious to find that an extremely belated effort to revive the study of astronomy, and at last to try to advance this

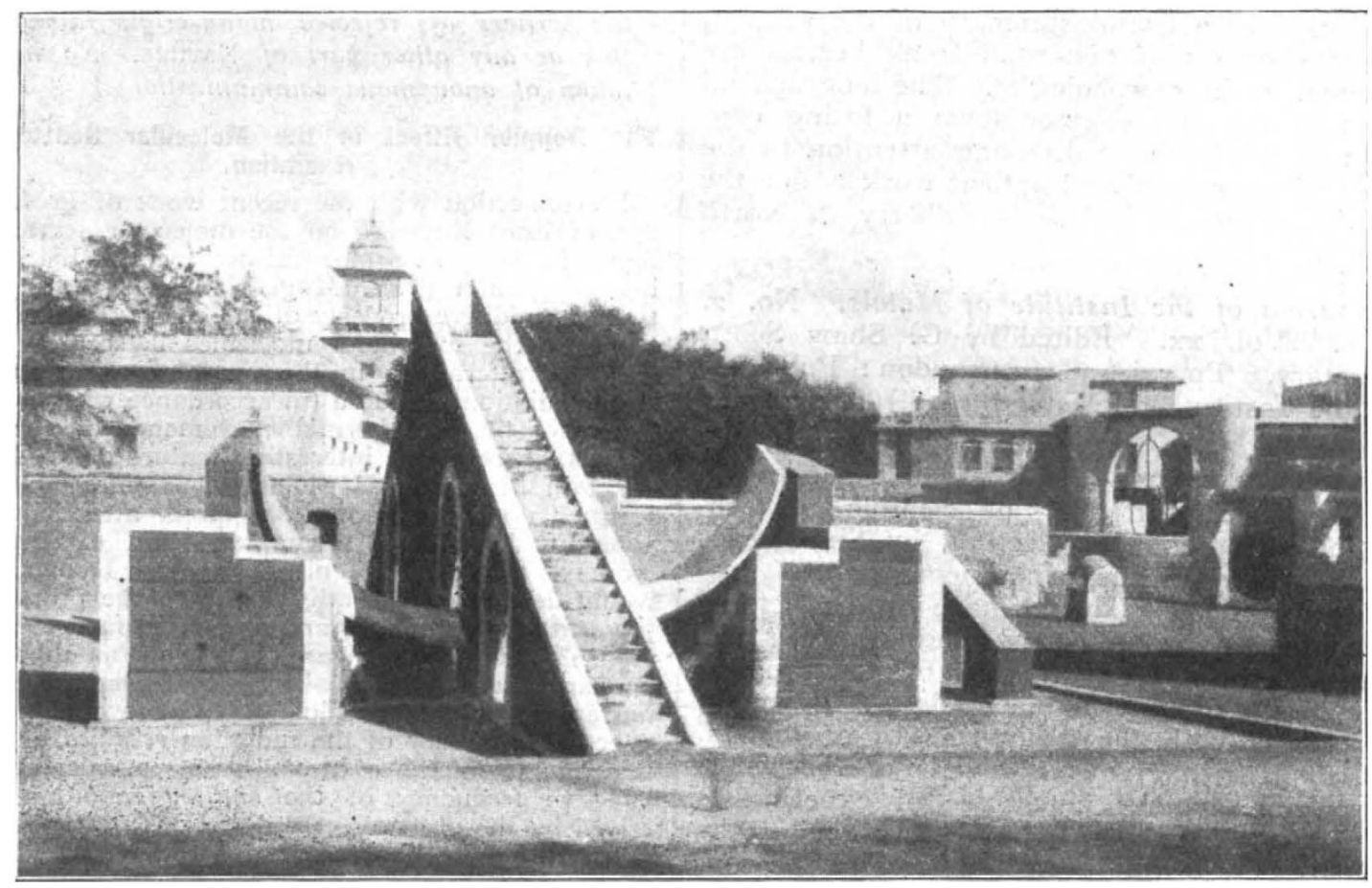

FIc. x.-General view, Jaipur Observatory. From "The Astronomical Observatories of Jai Singh."

effect. The specially favoured directions, for disturbances passed on by the molecules, must be almost coincident with the ray-must, in fact, belong to the ray after the manner of diffraction, when it is regarded as a physical filament of light rather than as a geometrical line.

The light that may be scattered in a crystal must, on any view, be due either to motes embedded in it or to the thermal motions of its molecules around their regularly spaced mean positions. A beautiful recent experiment by Prof. Strutt, now reported in the Proceedings of the Royal Society, exhibits a spiral beam scattered sideways when plane-polarised light passes through a column of quartz; thus, incidentally, it puts in direct visual evidence the slow rotation of the plane of polarisation of the vibrations which arises from the spiral structure of the crystal.

Cambridge, April 24. JUSEPH LARMOR. science by independent work, was made early in the eighteenth century in the north of India.

Rajah Jai Singh of Jaipur (born I686, died I743) was interested in astronomy from his youth. He wrote or caused to be written an astronomical work named after the Emperor Muhammad Shah, of which there are now extant an imperfect copy in Sanskrit (at Jaipur), and a complete MS. in Persian in the British Museum. It contains a star catalogue, which, however, is nothing but the catalogue of Ulugh $\mathrm{Beg}$, with $4^{\circ} 8^{\prime}$ added to the longitudes to allow for precession. A translation of the introduction was published by Hunter in the "Asiatic Researches," vol. v. (1799); it is

1 "The Astronomiçal Observatories of Jai Singh." By G. R. Kaye. (Archæological Survey of India, New Imperial Series, vol. xl.) Pp. viit+ $15 x+26$ plates +1 map. (Calcutta, $19 \times 8$.) Price 235 . 
reproduced by Mr. Kaye in the valuable book now under notice. In this introduction Hipparchus is referred to as an ignorant clown, and Ptolemy as a bat who can never arrive at the sun of truth; the demonstrations of Euclid are said to be an imperfect sketch, and "the European tables " to be often in error. Jai Singh therefore decided to erect new instruments of colossal size, similar to those made by Ulugh Beg at Samarkand in the fifteenth century. Among the latter we know from other sources that there was a quadrant of $180-\mathrm{ft}$. radius, while a $60-\mathrm{ft}$. sextant is said to have been erected at Baghdad in A.D. 992. Before the application of diagonal scales or verniers to graduated arcs there was only one way of making single minutes and fractions of them distinguishable-by enlarging the in- but gigantic sun-dials. Thus the Samrät Yantra, or "supreme instrument," consists of a gnomon in the form of a rectangular triangle with the hypotenuse parallel to the earth's axis, and an equatorial arc on either side. The two largest examples are at Delhi (height $68 \mathrm{ft}$., radius of $\operatorname{arcs} 49 \frac{1}{2} \mathrm{ft}$. ) and at Jaipur (90 ft. and $50 \mathrm{ft}$.) (Fig. I). The Jai Prakas is a hemispherical bowl, on the concave surface of which are marked the equator, meridian, and other circles, on which the shadows of wires might fall. The Ram Yantra (Fig. 2) is a cylindrical wall with a vertical pillar in the middle. Neither these nor any of the other instruments show any originality of design. The best known of these five collections of masonry instruments is probably the Delhi Observatory, called the Jantar Mantar, built about 1724 ,

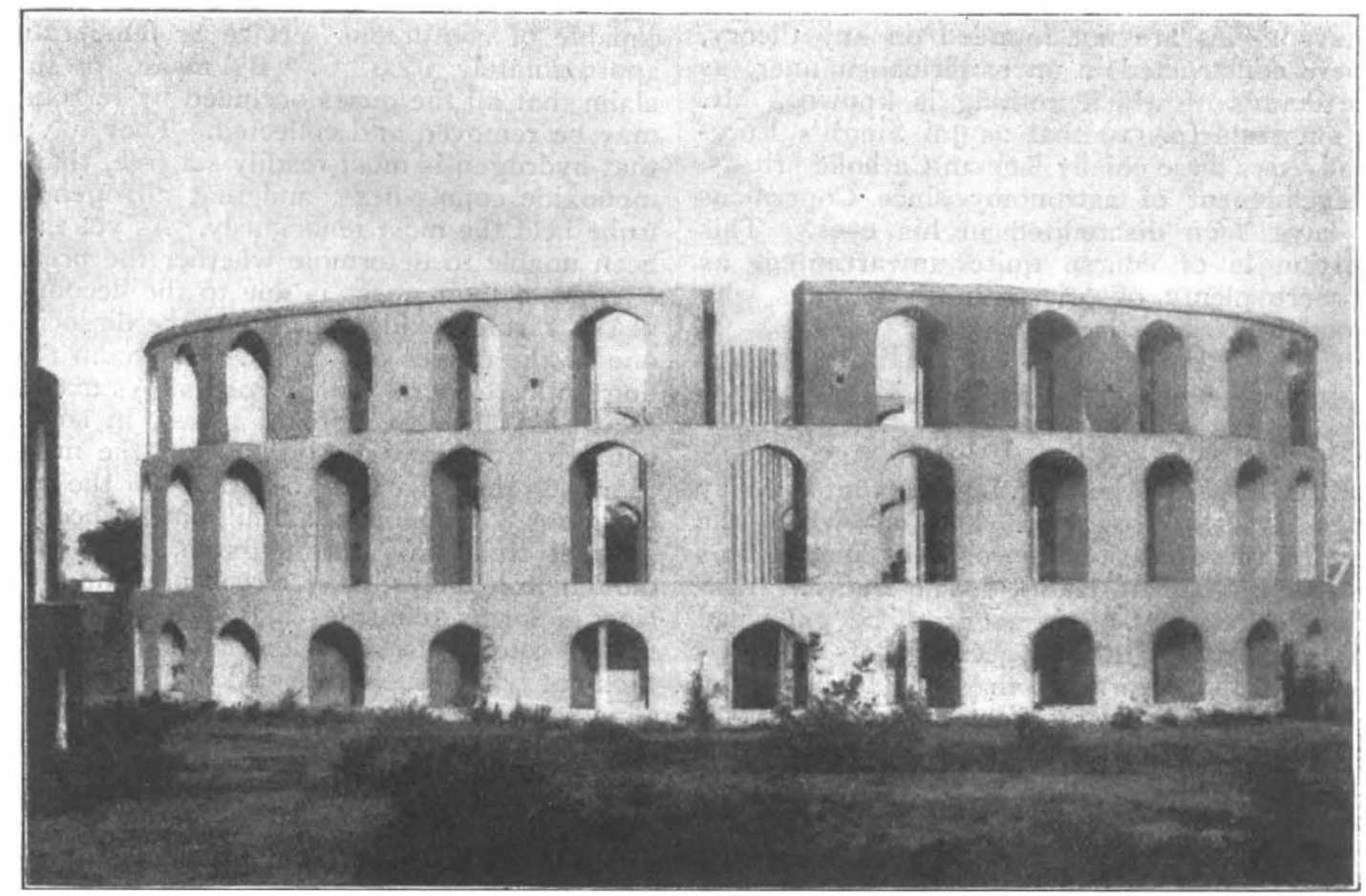

F1G. 2.-The Ram Yantra, Delhi, North Building. From "The Astronomical Observatories of Jai Singh."

struments as much as possible. Jai Singh first constructed a number of astrolabes of iron or brass, from 6 in. to $7 \mathrm{ft}$. in diameter, many of which are still preserved at Jaipur. In four plates Mr. Kaye gives sixteen photographic illustrations of these astrolabes, but most of them are too indistinct to show details.

Jai Singh's chief work, however, was the building of masonry instruments, ranging from a few feet to $90 \mathrm{ft}$. in height, at Delhi, Jaipur, Ujjain, Benares, and Mathura. They have often been described in a more or less sketchy manner in books on India; but $\mathrm{Mr}$. Kaye gives an exhaustive account of them, beautifully illustrated by a number of plates. ${ }^{2}$ They ane really nothing

2 There are a number of tiny models of them in the South Kensington Museum.

No. 2583 , VOL. IO3]
$3 \frac{1}{2}$ miles south of the Ridge, so that it will be a notable feature in the new capital. It contains six instruments. The graduations on the gnomon of the Samrāt Yantra are scratched into the limeplaster surface, but those of the quadrants are well marked with a soft, black stone neatly inlaid into the face of the arcs. This seems, however, to be the result only of a restoration carried out in I9ro-r2. At Jaipur the instruments are well preserved, being within the precincts of the palace. At Benares the instruments were erected in 1737 on the roof of a building; they are therefore of moderate size. A much-needed restoration took place in 1912. At Ujjain the four instruments are in a state of ruin, and those at Mathura have quite disappeared.

There is no record of any attempts having been 
made to do systematic work with these instruments, nor would it have been of the slightest use to employ them for anything but lecturedemonstrations. In 1728 or 1729 Jai Singh sent Figueredo, a Portuguese Jesuit, to Europe to procure astronomical tables, and he brought back "tables published under the name "Lir," i.e. the tables of La Hire. Mr. Kaye also thinks that Jai Singh possessed the "Historia Cœlestis" of Flamsteed, and says (p. 69) that "he must have been acquainted with the teaching of Kepler, Galilep, and Newton, for he possessed the works of La Hire, Flamsteed, and others." But neither of these works could give the slightest clue to the teaching of European astronomers. The "Historia Cœlestis " contains Flamsteed's observations and the resulting star-catalogue (as well as reprints of previous catalogues), and La Hire's planetary tables are not founded on any theory, but were constructed in an empirical manner, as to the details of which nothing is known. Mr. Kaye suggests (p. 90) that as Jai Singh's European advisers were chiefly Roman Catholic priests, the development of astronomy since Copernicus must have been discredited in his eyes. This suggestion is of course quite unwarranted, as there were plenty of priests in those days who did good work in astronomy.

\section{J. L. E. DREyer}

THE OCCLUSION OF GASES BY METALS. PRESIDING at the meeting of the Faraday 1 Society in November last, at which the subject of the occlusion of gases by metals was discussed, Sir Robert Hadfield delivered an introductory address, which is about to be published by the society with an account of the discussion. It appears from the bibliography attached to this address that Thomas Graham was one of the first to investigate this subject. His results were published in the Philosophical Transactions of the Royal Society in 1867 , the title of the paper being "The Occlusion of Gases by Meteoric Iron." The particular specimen investigated contained $90^{\circ} 9$.per cent. of iron, 8.45 per cent. of nickel, and a small quantity of cobalt. It was free from any stony admixture, and was remarkably pure and malleable. A strip cut from this with a clean chisel was first well washed with a hot solution of potash, then with distilled water, and afterwards dried. It was then placed in a porcelain tube which was evacuated and afterwards heated to redness in a combustion furnace.

Gas was observed to come off freely, and was collected in successive portions. The first portion evolved consisted principally of hydrogen. Succeeding portions also contained hydrogen as the chief constituent, with smaller quantities of carbonic oxide and nitrogen. This particular specimen of iron yielded $2 \cdot 8$ times its own volume of gas. Graham also investigated the gas taken up by iron from a carbonaceous fire, and in the case of some clean horseshoe nails which he heated in a similar way he found that the metal yielded 2.66 times its own volume of gas, containing about 50 per cent. of carbonic oxide, 35 per cent. of hydrogen, $7 * 7$ per cent. of carbon dioxide, and 7 per cent. of nitrogen. He concluded that as hydrogen was recognised in the spectrum analysis of the lights of the fixed stars, the Lenarto meteoric iron which he had investigated came from an atmosphere in which hydrogen was the chief constituent, and that it held imprisoned within it the hydrogen of the stars.

The latest investigations on the subject of "Occluded Gases in Ferrous Alloys" are those by Allemann and Darlington, whose results are published in the proceedings of the Franklin Institute of Philadelphia in February, March, and April of I9I8. These investigators describe a gastight vacuum furnace which they have constructed capable of continuous service at temperatures of approximately $1900^{\circ} \mathrm{C}$. By means of this they claim that all the gases occluded by ferrous alloys may be removed and collected. They have found that hydrogen is most readily set free, that carbon monoxide comes next, and that nitrogen appears to be held the most tenaciously. As yet they have been unable to determine whether the presence of oxygen in their gases is due to the decomposition of the various oxides of iron or the dissociation of one of the oxides of carbon. Allemann and Darlington have found that ferrous alloys may occlude relatively large volumes of gases, in some cases equal to 200 times the volume of the metal, and they suggest that, in addition to the ordinary functions of metals, aluminium, silicon, manganese, titanium, and tungsten, when added to molten iron alloys, may either prevent the occlusion of large quantities of gases or aid in eliminating such gases at lower temperatures than those at which such elimination ordinarily occurs. Finally, they have shown that the removal of these gases markedly changes the microstructure and increases the density of the alloys.

Sir Robert Hadfield then proceeds to consider the bearing of scientific work of the foregoing kind on the problem of obtaining sound steel, and quotes at some length the views of the late Dr. Héroult as set forth in his paper on "The Presence and Influences of Gases in Steel." Héroult pointed out that the gases obtained from blowholes in unsound steel ingots always contain hydrogen and nitrogen, often with only traces of carbon monoxide, but it is well known that sound steel, when heated in vacuo, also gives off these gases. It has also been shown that the quantities of gases so liberated are about the same, whether the steel be made by the crucible, the Bessemer, the open hearth, or the electric furnace. In consequence of this he concluded that hydrogen and nitrogen are not the cause of the production of blowholes, but that the latter are the result of the liberation of carbon monoxide, except in the case of blowholes near the surface, which are due to the poor condition of the moulds. Héroult's view of the production of blowholes was as follows :-

The carbon monoxide does not pre-exist in the NO. 2583 , voL. IO3] 